\title{
Title:
}

\section{Burning Mouth Syndrome - a diagnostic dilemma}

Richeal Ni Riordain $^{1,2}$, Sinead O’Dwyer ${ }^{3}$, Christine McCreary ${ }^{1}$

1. Cork University Dental School and Hospital, Wilton, Cork, Ireland

2. Eastman Dental Institute, London, UK

3. Dublin Dental University Hospital, Dublin, Ireland

Corresponding author:

Dr Richeal Ni Riordain

Honorary Clinical Lecturer in Oral Medicine

$+353214205012$

\section{richeal.niriordain@gmail.com}




\begin{abstract}
:
Background: Burning mouth syndrome (BMS) is a chronic pain disorder, more common in peri and postmenopausal females, with a varied symptomatology. Symptoms include a burning or stinging sensation of the tongue, lips or other oral mucosal surfaces, subjectively dry mouth or excess saliva, altered taste or lost of taste and paraesthetic sensations. These are usually present daily for more than 3 months.
\end{abstract}

Aims: The aims of this study were to highlight the symptomatic manifestations of BMS along with the need for prompt diagnosis and onward referral when necessary.

Methods: A cross-sectional study of patients with idiopathic BMS was conducted. The presenting symptoms, time to diagnosis and number of clinicians seen in advance of a diagnosis of BMS and anxiety and depression as determined by the Hospital Anxiety and Depression Scale (HADS) was recorded. Correlations were explored

Results: Fifty patients were enrolled in this study (38 F:12 M). The average time from onset of symptoms to diagnosis was 13 months. Commonly reported symptoms included burning $(n=44)$ and altered taste $(n=14)$. The median anxiety score was 13 and the median depression score was 10 . No statistically significant correlations were found between the anxiety and depression scores generated and the number of clinicians seen or the time to diagnosis.

Conclusion: The results of this study indicate that there is a need for an increased awareness of the symptoms reported in BMS, particularly in peri and post-menopausal women. This should aid prompt diagnosis and may alleviate some of the anxiety that patients may experience.

Keywords: Burning mouth syndrome, presenting symptoms, diagnostic delay 


\section{Introduction}

Burning mouth syndrome (BMS) is defined as 'an intraoral burning or dysaesthetic sensation, recurring daily for more than 2 hours per day over more than 3 months, without clinically evident causative lesions'[1]. Due to the emphasis on the absence of a clinically causative lesion the condition has been classified into primary BMS and secondary BMS. Secondary BMS is oral burning secondary to local or systemic causes[2]. Local factors can include candidiasis and salivary gland hypofunction whilst systemic factors may refer to diabetes mellitus and vitamin B12 deficiency for example[3]. Primary or idiopathic BMS is therefore considered to be a diagnosis of exclusion. In addition to oral burning, patients may also report subjectively dry mouth or excess saliva, altered taste or lost of taste and tingling or paraesthetic sensations[4]. Multiple terms are synonymous with BMS including oral dysaesthesia, glossodynia, glossopyrosis, stomatodynia, stomatopyrosis, sore tongue. This is a chronic pain disorder, typically described by patients as a burning or stinging sensation of the tongue, lips or other oral mucosal surfaces. With only $3 \%$ of patients experiencing spontaneous remission [5], effective management of this condition is of the utmost importance.

Diagnosing BMS can present a challenge for clinicians with Klasser et al referring to BMS as a diagnostic quandary [6]. Patients have often attended dentists, general practitioners (GPs), gastroenterologists and neurologists prior to diagnosis[7]. The diagnostic challenge presented by BMS can lead to diagnostic delay, with studies demonstrating an average delay of between 34 months and 41 months from first presentation to diagnosis $[8,9]$. Following the onset of symptoms this diagnostic delay may result in increased anxiety in patients. Using patientreported depression and anxiety scales, Gao et al found that patients with BMS had statistically significant higher depression and anxiety scores in comparison to age and sex matched healthy controls[7]. The aims of this study were to highlight the symptomatic manifestations of BMS along with the need for prompt diagnosis and onward referral when 
necessary. The objectives of this study were therefore to explore the presenting symptoms, time to diagnosis and number of clinicians seen in advance of a diagnosis of idiopathic BMS in a sample of patients newly diagnosed with BMS. A further investigation of correlations between anxiety and depression, measured using the Hospital Anxiety and Depression Scale, and the number of clinicians seen along with the time to diagnosis was also determined in the patient population.

\section{Methods}

A cross-sectional study of patients newly diagnosed with BMS attending the Oral Medicine Unit of Cork University Dental School and Hospital was conducted. All new patients referred to the Unit with symptoms including burning sensation, subjective xerostomia, oral paraesthesia, dysguesia, loss of taste and altered smell were screened for suitability. Screening included a full history, including a detailed drug history and medical history, a thorough clinical examination and appropriate laboratory evaluations to exclude any local or systemic cause for the symptoms reported as per the recommendations by Renton [3]. Due to the nature of the data being collected further exclusion criteria included a positive drug history of previous psychotropic medication, including antidepressants and anticonvulsants as these medications may influence the scores generated from anxiety and depression scale used. Patients with previous diseases associated with somatic symptoms, such as fibromyalgia and chronic backache were excluded again due to the influence these conditions would have on the HADS questionnaire.

Data collection was carried out by one of the study team (RNR) using a study specific data collection proforma, which included patient demographics, BMS symptoms experienced, time since onset of symptoms and clinicians seen prior to diagnosis. Patients also completed the HADS questionnaire, a valid and reliable tool used to detect anxiety and depression in outpatient populations [10]. HADS is a 14-item tool with each item scored on a 4-point frequency scale, ranging from not at all (0) to most of the time (3). Responses for the 7 
anxiety related items and 7 depression related items are summed independently, leading to the generation of 2 scores ranging from $0-21$. The higher the score, the more anxiety and or depression reported by the patients [11]. On the basis of their scores, individuals can be categorised into three score ranges that indicate the severity of the states; 'normal' (0-7), 'borderline abnormal' (8-10) and 'abnormal' (11-21).

Statistical Package for the Social Sciences (Version 24.0, SPSS Inc, Chicago, USA) was used to analyse the data. All skewed data were reported using median and interquartile range $\left[\mathrm{Q}_{1^{-}}\right.$ $\mathrm{Q}_{3}$ ] for sumarisation. As the data was mostly non-parametric Spearman Rho was calculated to determine the correlation anxiety and depression scores generated from HADS and the number of clinicians seen along with the time to diagnosis. Statistical significance was set at $\mathrm{p}<0.05$.

\section{Results}

A total of 50 patients were enrolled in this study, 38 of whom were female and 12 were male, with a mean age of 64 years (range from $34-83$ years). The average time since onset of symptoms was 13 months with a range of 1 to 60 months from onset to diagnosis of idiopathic BMS (median 6 months; interquartile range 6 - 24 months). Twenty-four patients had seen their dentist prior to referral, 13 had seen their GP, 3 patients had been seen in an Otorhinolaryngology outpatient clinic and 2 patients had been seen by a Gastroenterologist. A total of 9 patients had been seen by at least 2 clinicians prior to the diagnosis of idiopathic BMS.

Table 1 provides a summary of the presenting symptoms, with $20 \%$ of patients $(n=10)$ reporting 2 or more symptoms at the time of diagnosis. The most commonly reported symptoms included burning $(n=44)$ and altered taste $(n=14)$. Areas of the mouth where burning was experienced included the anterior and lateral aspects of the tongue, palate and lips. 
With regard to HADS the median anxiety score was 13 (interquartile range $7-17$ ) and the median depression score was 10 (interquartile range 5 - 16). Figure 1 demonstrates the number of patients in each of the HADS defined anxiety and depression severity states 'normal' (0-7), 'borderline abnormal' (8-10) and 'abnormal' (11-21). Correlations between anxiety and depression scores generated and the number of clinicians seen along with the time to diagnosis is represented in Table 3. No statistically significant correlations were found in this sample.

\section{Discussion}

Patients with BMS or oral dysaesthesia commonly present reporting persistent symptoms of intraoral burning or tingling, altered taste or a change in the perception of the amount of saliva in the mouth. With an unremarkable intraoral examination and whole unstimulated salivary flow rates within normal limits the local causes of these symptoms, such as candidiasis, can often be outruled. These symptoms may however also present as an oral manifestation of a systemic disease or as an adverse drug reaction. This makes the diagnosis of primary BMS more challenging. According to the Central Statistics Office in Ireland and Age UK the population is ageing $[12,13]$. With an ageing population it is anticipated that there will be a greater prevalence of patients with multi-morbidity and polypharmacy. In a review article regarding adverse drug reactions and the oral cavity by Yuan et al the authors highlight that medications are one of the most common causes of hyposalivation with dry mouth listed as an adverse drug event in over 500 medications [14]. Altered taste or dysgeusia can be associated with hyposalivation however it is also linked to a number of commonly used medications including ACE-inhibitors and proton pump inhibitors [15]. These oral symptoms may therefore become more commonplace with increasing polypharmacy and a thorough review of current medications is key in reaching a diagnosis of idiopathic BMS. 
Although epidemiological data regarding BMS is poor a recent study by Kohorst et al indicated that BMS is more common in females with a sharply increasing incidence after the age of 50 years [16]. Our study findings are in keeping with this data with an average age of 64 years and an approximate 3 to 1 ratio of females to males. As previously reported in the literature patients most commonly report intraoral burning of the lips and tongue [2] with some patient also presenting with dysgeusia and altered perception of intraoral salivary lubrication [17]. As these symptoms were present in our patient population for an average of over a year it can be expected that patients may turn to the Internet to explore potential diagnoses. Reassuringly when checking Google for causes of 'burning tongue and lips', the most common symptom reported in our patients, BMS is the first diagnosis offered. In a recent article evaluating the quality of web based material pertaining to the treatment of BMS the authors concluded that the reliability of the online information was questionable [18]. Although the initial diagnosis may be facilitated by 'Dr Google' the treatment options subsequently presented may be misleading for patients.

The aforementioned 13 month diagnostic delay may have contributed to the number of patients with 'abnormal' anxiety score according to HADS (60\%), with the median anxiety score of 13 recorded. This data must, however, be interpreted with caution as a high anxiety score in HADS does not confirm a diagnosis of generalised anxiety disorder. It is also known that anxiety and depression are more common in females, which represent the majority of this study sample [19]. A question that arises is whether increased anxiety and depression are primary or secondary events in BMS as chronic pain conditions can produce psychological disturbances. Distinct from generalised anxiety, health anxiety is characterised by the fear of having a serious disease in the absence a clinical confirmation of this grave illness [20]. This aspect of anxiety could add further to the challenge in managing patient with BMS as health anxiety may be exacerbated by the number of clinicians seen prior to a definitive diagnosis of idiopathic BMS. Grushka et al report that patient presenting with 'inexplicable oral complaints', such as those reported in BMS, are commonly referred to multiple clinicians 
with no effective treatment. As highlighted by the authors this may have a substantial emotional impact on patients, which could lead to health anxiety [21, 22]. Although anxiety and depression were evaluated in this study we did not explore health anxiety. This can be assessed using a tool such as the Health Anxiety Inventory (HAI), an 18 item tool developed by Salkovskis et al [23].

In conclusion, although BMS may only present in up to $3.7 \%$ of the population [17] an awareness of the symptomatic presentation is important to avoid the health service and economic burden of multiple consultation by numerous clinicians prior to a definitive diagnosis. Therefore in the clinical evaluation of patients presenting with burning of the tongue, lips or palate, isolated dysgeusia or subjective xerostomia BMS should be considered as part of the differential diagnosis. Onward referral to colleagues in Oral Medicine may expedite the diagnosis and management of patients with the chronic condition.

\section{Compliance with Ethical Standards}

Ethical approval: "All procedures performed in studies involving human participants were in accordance with the ethical standards of the institutional and/or national research committee and with the 1964 Helsinki declaration and its later amendments or comparable ethical standards."

Conflict of Interest: The authors declare that they have no conflict of interest.

Informed consent: "Informed consent was obtained from all individual participants included in the study." 


\section{References}

1. Headache Classification Committee of the International Headache, S., The International Classification of Headache Disorders, 3rd edition (beta version). Cephalalgia, 2013. 33(9): p. 629-808.

2. Scala, A., et al., Update on burning mouth syndrome: overview and patient management. Crit Rev Oral Biol Med, 2003. 14(4): p. 275-91.

3. Renton, T., Burning Mouth Syndrome. Rev Pain, 2011. 5(4): p. 12-7.

4. Speciali, J.G. and J. Stuginski-Barbosa, Burning mouth syndrome. Curr Pain Headache Rep, 2008. 12(4): p. 279-84.

5. Sardella, A., et al., Burning mouth syndrome: a retrospective study investigating spontaneous remission and response to treatments. Oral Dis, 2006. 12(2): p. 152-5.

6. Klasser, G.D., J.B. Epstein, and D. Villines, Diagnostic dilemma: the enigma of an oral burning sensation. J Can Dent Assoc, 2011. 77: p. b146.

7. Gao, J., et al., A case-control study on etiological factors involved in patients with burning mouth syndrome. J Oral Pathol Med, 2009. 38(1): p. 24-8.

8. Mignogna, M.D., et al., The diagnosis of burning mouth syndrome represents a challenge for clinicians. J Orofac Pain, 2005. 19(2): p. 168-73.

9. Klasser, G.D., et al., Burning mouth syndrome: a challenge for dental practitioners and patients. Gen Dent, 2011. 59(3): p. 210-20; quiz 221-2.

10. Bjelland, I., et al., The validity of the Hospital Anxiety and Depression Scale. An updated literature review. J Psychosom Res, 2002. 52(2): p. 69-77.

11. Snaith, R.P., The Hospital Anxiety And Depression Scale. Health Qual Life Outcomes, 2003. 1: p. 29.

12. Age UK, Later life in the United Kingdom. 2016, Age UK: London. 
13. Central Statistics Office Ireland. Age Proflie of Ireland. 2017 [cited 2018 March 12th].

14. Yuan, A. and S.B. Woo, Adverse drug events in the oral cavity. Oral Surg Oral Med Oral Pathol Oral Radiol, 2015. 119(1): p. 35-47.

15. Tuccori, M., et al., Drug-induced taste and smell alterations: a case/non-case evaluation of an italian database of spontaneous adverse drug reaction reporting. Drug Saf, 2011. 34(10): p. 849-59.

16. Kohorst, J.J., et al., A population-based study of the incidence of burning mouth syndrome. Mayo Clin Proc, 2014. 89(11): p. 1545-52.

17. Bergdahl, M. and J. Bergdahl, Burning mouth syndrome: prevalence and associated factors. J Oral Pathol Med, 1999. 28(8): p. 350-4.

18. Alnafea, S., et al., Online Information on the Treatment of Burning Mouth Syndrome: Quality and Readability. J Oral Facial Pain Headache, 2017. 31(2): p. $147-151$.

19. Zender, R. and E. Olshansky, Women's mental health: depression and anxiety. Nurs Clin North Am, 2009. 44(3): p. 355-64.

20. Sunderland, M., J.M. Newby, and G. Andrews, Health anxiety in Australia: prevalence, comorbidity, disability and service use. Br J Psychiatry, 2013. 202(1): p. 56-61.

21. Grushka, M., J.B. Epstein, and M. Gorsky, Burning mouth syndrome. Am Fam Physician, 2002. 65(4): p. 615-20.

22. Aggarwal, A. and S.R. Panat, Burning mouth syndrome: A diagnostic and therapeutic dilemma. J Clin Exp Dent, 2012. 4(3): p. e180-5. 
23. Salkovskis, P.M., et al., The Health Anxiety Inventory: development and validation of scales for the measurement of health anxiety and hypochondriasis. Psychol Med, 2002. 32(5): p. 843-53. 